Revue

Revue de l'histoire des religions

de Ihistoire des religions

$4 \mid 2008$

Varia

Jean-Marie SALAmito, Les Virtuoses et la Multitude. Aspects sociaux de la controverse entre Augustin et les pélagiens

Charlotte Touati

(2) OpenEdition

Journals

Édition électronique

URL : http://journals.openedition.org/rhr/7003

DOI : $10.4000 /$ rhr.7003

ISSN : 2105-2573

Éditeur

Armand Colin

Édition imprimée

Date de publication : 1 décembre 2008

Pagination : 550-554

ISSN : 0035-1423

Référence électronique

Charlotte Touati, « Jean-Marie salamito, Les Virtuoses et la Multitude. Aspects sociaux de la controverse entre Augustin et les pélagiens », Revue de l'histoire des religions [En ligne], 4 | 2008, mis en ligne le 15 janvier 2010, consulté le 22 septembre 2020. URL : http://journals.openedition.org/rhr/ 7003 ; DOl : https://doi.org/10.4000/rhr.7003

Ce document a été généré automatiquement le 22 septembre 2020.

Tous droits réservés 


\title{
Jean-Marie SALAMITO, Les Virtuoses et la Multitude. Aspects sociaux de la controverse entre Augustin et les pélagiens
}

\author{
Charlotte Touati
}

\section{RÉFÉRENCE}

Jean-Marie SALAMITo, Les Virtuoses et la Multitude. Aspects sociaux de la controverse entre Augustin et les pélagiens, Grenoble, Éditions Jérôme Millon, 2005, $22 \mathrm{~cm}, 351 \mathrm{p}$.

(« Nomina »), $29 €$.

1 S'il n'était le sous-titre, «les virtuoses et la multitude" serait une formule plutôt obscure. Ainsi éclairée, elle se révèle fort judicieuse pour qualifier non seulement la problématique de l'étude de Jean-Marie Salamito, c'est-à-dire le discours sur la position de l'élite religieuse face au tout-venant des fidèles, mais aussi la méthode permettant d'aborder cette même problématique. Dans cette étude, tout est question d'approche, de représentation et de discours: discours du cinquième siècle conservé dans les œuvres d'Augustin, de Pélage et de Julien d'Éclane, discours du vingtième siècle, celui de Max Weber notamment.

Inspiré par les travaux du fameux sociologue sur la Réforme et sa diffusion selon des contingences économiques et culturelles (Die protestantische Ethik und der Geist des Kapitalismus [1904-1905]), J.-M. Salamito en réutilise les catégories, ainsi que le vocabulaire, pour les appliquer à la querelle pélagienne. Le lecteur comprendra rapidement que « virtuoses » est un terme technique réservé aux fidèles vivant selon le plus haut degré d'exigence de la doctrine qu'ils ont embrassée. Ce vocable fut initialement forgé pour qualifier les tenants de certaines «sectes » nord-européennes $\mathrm{du}$ seizième siècle. J.-M. Salamito se propose de l'appliquer à une réalité 
méditerranéenne du cinquième siècle. Ce saut hardi, mais réussi, à travers le temps démontre l'efficacité du modèle wébérien, qui peut dès lors prétendre à une certaine universalité. Universalité relative, bien entendu, puisque le cadre général reste celui de la religion chrétienne et de l'héritage gréco-romain.

3 Que le lecteur se garde d'attendre un dialogue par-dessus les siècles: J.-M. Salamito emprunte la méthode de $\mathrm{M}$. Weber, mais non son sujet. Le propos est donc de cerner la crise pélagienne ainsi que certains points de doctrine des protagonistes avec des outils nouveaux.

De la fameuse «crise pélagienne » subsistent les textes des deux partis en présence, ce qui est une aubaine sachant que la plupart des querelles doctrinales est documentée du seul côté du vainqueur. Les sources les plus connues sont sans aucun doute les œuvres d'Augustin, dont J.-M. Salamito a une connaissance approfondie. Pour établir le dossier, ce n'est pas moins que l'ensemble du corpus augustinien qui a été dépouillé. Les textes faisant état d'attaques directes contre Pélage ont été retenus en priorité, mais, s'inscrivant dans une démarche polémique, ils n'échappent pas à quelques outrances et autres raccourcis. Pour connaître les motifs qui poussèrent Augustin à s'investir personnellement dans le débat à travers lettres, sermons et traités, il convient de s'intéresser aussi à ses positions de principe sur la grâce, le libre arbitre, le baptême ou les engagements de vie. J.-M. Salamito ne propose cependant pas un abrégé de la doctrine augustinienne; les renvois bibliographiques sont suffisamment clairs pour orienter le lecteur, sans désagréger le fil de l'argumentation. Quelques cas concrets sont privilégiés, à titre d'illustrations, notamment la correspondance entretenue autour de la prise de voile de Démétriade, jeune aristocrate romaine. L'exemple est exceptionnel car il traduit ce qui pourrait rester querelle de théologiens dans une réalité, celle de la conduite spirituelle d'une jeune femme. Elle prend ici la forme d'un double dialogue ou d'un échange indirect entre Augustin et Pélage, dont l'enjeu, autant que le prétexte, est Démétriade. Précieux trophée.

5 La première partie des Virtuoses et la Multitude est consacrée à ce dossier, ce qui permet d'asseoir l'argument de son auteur: Pélage et Augustin se réjouissent aussi bien l'un que l'autre du choix de Démétriade et l'exhortent à maintenir la droiture de la voie qui est sienne à présent. Ils tiennent cependant des discours divergents et celui de Pélage intéresse J.-M. Salamito au premier chef. Il y décèle un langage aristocratique, celui des "virtuoses", taillé pour seoir au patriciat de l'empire. En effet, selon la harangue pélagienne, c'est en souvenir de ses nobles origines que Démétriade est, et doit rester, une chrétienne d'exception. À cet élitisme déclaré, Augustin oppose un discours d'humilité, sans doute plus familier au chrétien du vingt-et-unième siècle, et adressé à la masse. J.-M. Salamito constate que chacun des deux théologiens a trouvé son public et ses mots. Le philologue n'en reste toutefois pas à cette conclusion empirique. On le constate en descendant dans les structures de la première partie.

6 Les chapitres II à IV voient apparaître le cache à l'aide duquel va être décryptée la doctrine de chacun des protagonistes. La base théorique est celle des "affinités électives» mises en œuvre par M. Weber dans son Éthique du Protestantisme. En un temps et un lieu donnés, il s'agit de l'adéquation des valeurs et de l'idéal vers lesquels tend un groupe social avec ce que propose une religion, une doctrine, voire une idéologie. Ainsi y aurait-il superposition du paradigme suivi par le noble romain et celui du moine pélagien: tous deux élitistes, "oisifs", guerriers et avides de reconnaissance. De même, les injonctions d'humilité, de discrétion et les 
« compensations spirituelles » entrevues par Augustin dans l'au-delà pour le fidèle qui aura su souffrir en ce monde trouveraient davantage d'écho dans la réalité de la plèbe.

7 Par un effet de balancier, les chapitres V et VI cherchent à gommer ce que les précédents montraient de trop mécaniste. Il s'agit du classique morceau de pondération visant à rééquilibrer les chapitres d'ouverture. Mais à trop vouloir nuancer la grille de lecture mise en place, l'auteur la fragilise au point que le lecteur n'ose plus s'en servir. L'auteur fournit lui-même des arguments à ses (éventuels) détracteurs en soulignant les contradictions inhérentes à son corpus.

Ce point de l'étude est à la fois heureux et autodestructeur. D'une part, il apporte sa touche au tableau d'ensemble en étendant l'analyse (pélagianisme élitiste vs augustinisme plébéien) à des groupes humains entiers, tandis que les quatre premiers chapitres étaient focalisés sur l'individu et sa conscience. D'autre part, il se conclut par un paragraphe intitulé « Entre aristocratisation du christianisme et démocratisation de la culture » : un modèle de consensus. L'élitisme de Pélage est en fait désamorcé par la vulgarisation de l'idéal aristocratique vers lequel il tend. La mitigation des exigences le met à la portée d'un plus grand nombre. C'est le second coup dur qui frappe cette démonstration cherchant à souligner les affinités électives entre le courant pélagien et l'aristocratie romaine. Mais si l'argument du livre s'essouffle, l'auteur trouvera-t-il les ressources pour dynamiser les quelque cent cinquante pages à suivre? Contre toute attente, oui.

Non sans une certaine perplexité, le lecteur entame la seconde partie et fait la connaissance d'un nouveau protagoniste : Julien d'Éclane. Les thèses qu'il développe ne sont que partiellement nouvelles, puisqu'elles émanent d'un fervent partisan de Pélage, mais elles innovent tout de même... par leur outrance. L'intransigeant disciple, issu de la noblesse italienne, a une très haute idée de ses origines et de son éducation classique. Inutile de préciser que de tels fondements s'éloignent sensiblement de l'idéal du moine breton. En face, Augustin, toujours. Si J.-M. Salamito semblait quelque peu douter de l'efficience de sa grille d'analyse, force est de constater un regain de confiance dans cette seconde partie. Il s'investit même personnellement au point de laisser affleurer son sentiment sur Julien et Augustin, chacun devenant le symbole de positions bien arrêtées. Arrêtées par l'auteur également.

10 Les textes sont toujours très présents, qu'il s'agisse de traités ou au contraire des pamphlets et ouvrages de circonstance rédigés au plus fort de la querelle opposant les deux évêques. Le message qu'ils transmettent, en revanche, reste tributaire des choix de mise en scène de J.-M. Salamito. Dans Les Virtuoses et la Multitude, Julien d'Éclane endosse le rôle de l'aristocrate suffisant ne côtoyant que des philosophes, regardant la foule avec morgue et reprochant à Augustin de manipuler celle que lui-même n'a pas su conquérir. Augustin, en évêque populaire, incarne le bon sens et siffle la fin de la pause lorsque les diatribes de Julien offusquent par trop la pensée chrétienne. Parfois, la critique morale affleure sous le commentaire littéraire et brouille légèrement la perspective historique : observe-t-on la bataille selon le point de vue du christianisme au quatrième ou au vingtième siècle?

11 L'image est rapidement restaurée, le chapitre $\mathrm{X}$ nous rappelant sans conteste à l'Antiquité tardive et à une spécificité de la querelle pélagienne: la question de la prédestination. Le salut pour un petit nombre d'élus : y a-t-il quelque élitisme dans la position d'Augustin en mesure de séduire les pélagiens? Non, puisque ni la naissance, ni le mérite gagné par l'ascèse et la discipline (signes distinctifs du pélagianisme) ne 
peuvent garantir la rédemption. Tout au plus engendrent-ils autosatisfaction et bonne conscience. Au contraire, la prédestination selon le modèle augustinien, secrète et mystérieuse, laisse le chrétien en suspens, livré à son Dieu. Elle le maintient dans l'inquiétude et l'espérance, germes d'humilité.

12 Ces ultimes considérations ne sont-elles pas justement celles des seizième et dixseptième siècles? S'il n'était quelques soupçons d'anachronisme, nous pourrions conclure qu'il existe des "affinités électives » entre le microcosme étudié par J.-M. Salamito et la doctrine pour laquelle Max Weber a forgé ses outils. Les écrits d'Augustin servent probablement d'interface entre l'Afrique du cinquième siècle et certains milieux protestants, qui s'abreuvèrent sans réserve à la source augustinienne. Un effet parasite dû à des emprunts directs n'est donc pas exclu. Il n'en reste pas moins intéressant de constater qu'un outillage mental a été élaboré à partir de la pensée d'un évêque défunt depuis des siècles, que ce même outillage a été ultérieurement analysé par un sociologue pour fonder sa méthode, et que cette méthode est finalement appliquée aux textes initiaux de l'évêque d'Hippone. Juste retour des choses.

13 J.-M. Salamito a certainement désiré faire passer son intuition géniale de l'état de sentiment subjectif et empirique à celui d'étude systématique et documentée. Les Virtuoses et la Multitude est un éclair, la connexion violente et précise entre deux univers (Réforme/Antiquité tardive), suivi d'une démonstration pesante, comme si l'auteur cherchait à atténuer la fulgurance de son trait. À trop vouloir démontrer, J.-M. Salamito se perd et prête le flanc à la critique. Quelques essentiels font défaut: les critères mis en œuvre dans le choix des textes, ou le rappel clair des catégories wébériennes qui apparaissent en cours d'étude et supposent la consultation des œuvres du sociologue. On ne peut que déplorer aussi une certaine répétitivité. À travers les premiers chapitres, le lecteur comprend que la doctrine pélagienne rejoint les valeurs de l'aristocratie romaine, que son discours sur l'excellence est détourné par une habile interpretatio christiana en une exhortation à l'ascèse, loin de la multitude. Loin de cette plebs, dont Augustin devient le champion au point de frayer avec le populisme. Mais pour prévenir l'infamie d'un tel terme, J.-M. Salamito introduit la notion de « christianisme moyen ». Il s'agirait d'un idéal démocratisé, mis à la portée de tous sans déterminisme social.

14 Au final, les prémisses exposées en quelques pages tiennent lieu de conclusion : la doctrine pélagienne, essentiellement fondée sur l'éthique, la discipline et la rigueur, affronte celle d'Augustin, davantage marquée par les concepts de foi et de grâce. Tout le reste n'est que démonstration, exemple, mais aussi contre-exemple. Malgré quelques répétitions, Les Virtuoses et la Multitude n'en reste pas moins une étude des plus stimulantes, tant au niveau du projet intellectuel que de la base documentaire. Érudition et hardiesse y président. Celle-ci a pu choquer celle-là, et l'auteur se serait senti obligé de produire tout son matériel, de pondérer son propos et de préserver, voire de conforter, l'image d'Augustin. 


\section{AUTEURS}

\section{CHARLOTTE TOUATI}

Université de Lausanne 